\title{
Genetic Mutation Analysis in Small Cell Lung Cancer by a Novel NGS-Based Targeted Resequencing Gene Panel and Relation with Clinical Features
}

\author{
Wang Jin $\mathbb{D D}^{1}{ }^{1}$ Zhao Lei, ${ }^{1}$ Sun $\mathrm{Xu},{ }^{2}$ Zhou Fachen, ${ }^{1}$ Zhang Yixiang, ${ }^{1}$ Zhao Shilei, ${ }^{1}$ Guo Tao, ${ }^{1}$ \\ Sun Zhe, ${ }^{1}$ Li Fengzhou, ${ }^{1}$ Wen-Hui Su, ${ }^{3}$ and Gu Chundong ${ }^{1}$ \\ ${ }^{1}$ Department of Thoracic Surgery, The First Affiliated Hospital of Dalian Medical University, Dalian, Liaoning 116011, China \\ ${ }^{2}$ Department of Pathology, The First Affiliated Hospital of Dalian Medical University, Dalian, Liaoning 116011, China \\ ${ }^{3}$ Department of Biomedical Sciences, Graduate Institute of Biomedical Sciences, College of Medicine, Chang Gung Molecular \\ Medicine Research Center, Chang Gung University, Taiwan
}

Correspondence should be addressed to Gu Chundong; guchundong@dmu.edu.cn

Received 27 June 2020; Revised 17 December 2020; Accepted 13 January 2021; Published 7 April 2021

Academic Editor: Fernando Toshio Ogata Copyright () 2021 Wang Jin et al. This is an open access article distributed under the Creative Commons Attribution License, which
permits unrestricted use, distribution, and reproduction in any medium, provided the original work is properly cited.

\begin{abstract}
Background. Small cell lung cancer (SCLC) is an aggressive and invasive malignancy that presents at advanced clinical stage with no more effective treatments. Development of a method for its early detection would be useful, also new therapeutic target need to be discovered; however, there is a lack of information about its oncogenic driver gene mutations. Objectives. We aim to identify the SCLC-related genomic variants that associate with clinical staging and serum protein biomarkers observed in other types of lung cancer. Methods. We screened formalin-fixed paraffin-embedded (FFPE) biopsy tissues of 32 Chinese SCLC patients using the 303 oncogenic driver gene panel generated by Tiling PCR amplification sequencing (tPAS) and analyzed the patients' corresponding serum protein levels of CYFRA21-1 CEA, NSE, and SCCA. Results. In total, we found 147 SCLC-related mutant genes, among these, three important genes (TP53, RB1, KMT2D) as well as five novel genes LRRK2, BRCA1, PTCH1, ARID2, and $A P C$ that altogether occurred in $90 \%$ of patients. Furthermore, increased mutations to 6 genes (WT1, NOTCH1, EPHA3, KDM6A, SETD2, ACVR1B) significantly associated with higher serum NSE levels $(P=0.0016)$ and higher clinical stages II + III compared to stage I $(P=0.06)$. Conclusions. Our panel is relatively reliable in detecting the oncogenic mutations of Chinese SCLC patients. Based on our findings, it may be possible to combine SCLC-related mutations and serum NSE for a simple detection of clinical staging.
\end{abstract}

\section{Background}

Lung cancer is a major cause of cancer-related deaths worldwide $[1,2]$. Small cell lung cancer $(S C L C)$, accounting for approximately $15 \%$ of lung cancers [3], is an aggressive, neuro-endocrine tumor characterized by a short doubling time, high growth rate, and early onset of metastases [4, 5]. While treatment includes surgery, chemotherapy and radiotherapy [6], the majority of patients present at advanced stages with loco-regional and systemic involvement and have poor prognosis and limited treatment options. Thus, a method for the early detection of SCLC is required and new treatments' targets need to be discovered.

There are no specific protein biomarkers identified for the SCLC. However, there are growing reports of a number of potentially useful serum proteins for the diagnosis of lung, prostate, and colorectal cancer [7-9]. Significantly, shorter survival rates have been associated with high levels of circulating tumor biomarkers, e.g., cytokeratin-19 fragment, neuron-specific enolase (NSE), and thymidine kinase, in patients with non-small-cell lung carcinoma (NSCLC) [10]. Tumor biomarkers can be further combined with certain 
mutations to improve the sensitivity for the detection of cancers, such as the carcinoembryonic antigen (CEA), carbohydrate antigen 19-9 (CA19-9), osteopontin, and hepatocyte growth factor that are combined with KRAS mutation to detect pancreatic cancer [11].

Due to the low incidence and survival rates from SCLC [12], as well as difficulty in obtaining sufficient tissue samples for research [13], still not enough is known about the driver mutations that associate with cancer development and progression. So far, exome sequencing of SCLC has highlighted the important mutations in the TP53, RB1, and histonemodifying genes [14]. Inactivating mutations in TP53 and $R B 1$ are found in $65-90 \%$ of SCLC patients [14-17]. Truncating frameshift, splice, and nonsense site variants in the KMT2D, which encodes a histone $\mathrm{H} 3 \mathrm{~K} 4$ methyltransferase, are found in $8 \%$ of SCLC tumors and $17 \%$ of SCLC cell lines [18].

To increase our understanding of the mutational signatures of SCLC and assist with development of new diagnostic tools, we analyzed the formalin fixed and paraffin embedded (FFPE) biopsy specimens of 32 Chinese SCLC patients with a new technology called Tiling PCR amplification sequencing (tPAS) based on the sequencing of multiplex PCR amplicons, which can simultaneously genotype single nucleotide variants (SNVs), insertions or deletions (indels), and copy number variations (CNVs) in a panel of 303 cancer genes, selected from the Catalogue of Somatic Mutations in Cancer [19], Cancer Genome Atlas [20,21], and Oncomine database [22].

\section{Materials and Methods}

2.1. Study Participants. We recruited 32 patients diagnosed with SCLC into this study. The tumor specimens were obtained from patients by surgeons in the Oncology Department of The First Affiliated Hospital of Dalian Medical University. All participants gave written informed consent for the provision of clinical information, biospecimen collection, and analysis. This study was approved by the Dalian Medical University and Hospital Research Ethics Committee. The Institutional Review Board (IRB) for this study is YJ-JGQX-2018-146.

2.2. Pathological Analysis of Tumor Specimens. Tumor specimens were acquired by surgery ( $>2 \%$ of total tissue mass and $>150$ cells). Diagnosis of SCLC was confirmed by pathologists using histologic evaluation of the stained FFPE tumor sections. TNM staging system of International Association for the Study of Lung Cancer (version 7) was used to determine the clinical staging.

2.3. Extraction of DNA from Formalin Fixed Paraffin Embedded (FFPE) Biopsy Specimens. Macrodissection was performed to increase the tumor tissue percentage to $\sim 80 \%$ prior to DNA extraction. Five to ten FFPE sections $(5 \mathrm{~mm}$ thickness) of each tumor specimen were used to prepare the genomic DNA for mutation profiling. DNA was extracted from FFPE tissue using the AmoyDxò FPPE DNA Kit and DNA purification spin columns (Amoy, Xiamen, Fujian). All purified DNA samples were determined to be of high quality by spectroscopy analysis and were suitable for mutation analysis by the tPAS assay.

2.4. Targeted Next-Generation Sequencing (NGS). Targeted NGS was performed on the 32 FFPE samples. Libraries were prepared according to Paragon Genomics manufacturer's protocol. Briefly, $40 \mathrm{ng}$ of human DNA was used for each multiplex PCR reaction. CleanPlex ${ }^{\mathrm{TM}}$ panels were supplied with $2 \mathrm{x}$ concentrated primer pools. Using thin-wall PCR strip tubes, components were added to run the PCR program for amplify target DNA regions. For panels consisting of multiple primer pools, $10 \mu \mathrm{l}$ multiplex PCR reactions were combined for each sample. Magnetic bead suspensions were vortexed vigorously, and $1.3 \mathrm{x}$ sample volume of magnetic bead suspension was added to each sample. After beads were drawn onto one side of the wall, the supernatant was removed and discarded without touching the beads, $10 \mu \mathrm{l}$ TE buffer was added to each tube, and then, the DNA was immediately released from the beads. Amplicons were purified, and concentration of the library was measured.

2.5. Tiling PCR Amplification Sequencing (tPAS). Paired-end $2 \times 150$ base pairs (bp) reads were generated from the Amplicon libraries using NextSeq CN500. Adaptor and low-quality bases in raw reads were trimmed using flexbar version v2.5 and aligned to the human reference genome hg19 using BWA-MEM version 0.7.5a. GATK v3.6 was used for local indel realignment and base quality recalibration [23]. GATK MuTect2 was used in somatic mutation (single nucleotide variants and small insertions and deletions) calling [24]. Somatic variant loci were defined by loci that were covered by $\geq 100$ total reads and supported by $\geq 4$ variant reads in the tumor and in the matched normal sample covered by $\geq 50$ total reads and supported by $\leq 2$ variant reads. ANNOVAR and TransVar were used for annotation with public variant databases $[25,26]$. Variants were filtered if the baseline population frequency $\geq 5 \%$.

2.6. Statistical Analysis. Nonparametric statistical tests were used throughout analysis due to a small sample size, and where $P$ values were determined, the significance level was set at 0.05 . Data for qualitative variables were reported as median and range. The association of clinical stage with clinical values, i.e., age and serum levels of protein biomarker, was analyzed as a continuous variable using the Wilcoxon rank sum test, while sex was analyzed as a categorial variable using the Fisher's exact test. The association between NSE levels and mutation counts was assessed using Spearman's correlation test. Statistical analysis was performed using $\mathrm{R}$ version 3.6.0.

\section{Results}

3.1. Baseline Characteristics. Table 1 shows the clinical characteristics of 32 Chinese SCLC patients. They tended to be older, at 65 years of age, and male. Under TNM classification, T2 and N0 were the most common categories (Table S1). We further classified patients into two broad clinical staging groups, stage I $(n=16)$ versus stages II + III $(n=16)$, to examine whether there were statistical differences between 
TABLE 1: Clinical characteristics of SCLC patients.

\begin{tabular}{|c|c|c|c|c|}
\hline Parameters & Clinical values* & Stage I & Stages II + III & $P$ value** \\
\hline Age (years old) & $65(49,81)$ & $67(51,81)$ & $63(49,81)$ & 0.2506 \\
\hline Sex (male/female) & $24 / 8$ & $12 / 4$ & $12 / 4$ & 0.9999 \\
\hline CYFRA21-1 (ng/ml) & $4.94(1.24,19.53)$ & $3.41(1.24,19.53)$ & $3.07(2.04,4.43)$ & 0.5033 \\
\hline CEA (ng/ml) & $4.33(0.83,18.37)$ & $2.97(0.83,16.59)$ & $2.52(1.37,18.37)$ & 0.9786 \\
\hline NSE (ng/ml) & $14.37(1.83,34.00)$ & $10.74(1.83,20.26)$ & $15.60(11.38,34.00)$ & $7.56 \times 10^{-4}$ \\
\hline SCCA (ng/ml) & $0.97(0.20,3.08)$ & $0.88(0.20,1.83)$ & $0.86(0.21,3.08)$ & 0.9742 \\
\hline
\end{tabular}

${ }^{*}$ Reported are median and range, except for sex is total individuals. ${ }^{* *} P$ values for age, CYFRA21-1, CEA, NSE, and SCCA were generated by Wilcoxon rank sum test; $P$ value for sex was generated by Fisher's exact test.

cancer stage and clinical values. Among the four investigated serum protein biomarkers, we found that serum levels of NSE of patients at stages II + III were significantly higher compared to stage I $\left(P=7 \times 10^{-4}\right.$; Table 1 and Figure S1).

3.2. Identification of SCLC-Related Oncogenic Genes. An overview of this descriptive case study is shown in Figure 1(a). From the 32 SCLC patients, we detected a total of 147 oncogenes. Each patient harbored an average of 12.6 (range: 3, 90) pathogenic mutations. Total mutations and genes across patients arranged by clinical stage are shown in Figure 1(b). Table S2 shows the list of 147 SCLC-related genes ranked by total mutation counts. After we ranked the genes according to mutation number, we found TP53, RB1, and KMT2D were the most frequently mutated genes (representing top $2 \%$ of all genes, observed in $79 \%$ of patients). Including five more genes that shared same number of mutations, LRRK2, BRCA1, PTCH1, ARID2, and $A P C$, increased the representation to top $5 \%$ of all genes, observed in $90 \%$ of patients. Together, these eight genes were the cut-off for the commonly mutated genes in this Chinese population of SCLC patients (Figure 1(c)).

3.3. Profiling of SNVs, Indels, and CNVs. The most common nucleotide mutations to the genes TP53, RB1, and KMT2D were $\mathrm{C}>\mathrm{A}, \mathrm{C}>\mathrm{T}$, and $\mathrm{C}>\mathrm{A}$ transitions, respectively (Figure 1(c)). Total number of mutations per coding area of a tumor genome (tumor mutational burden (TMB)) ranged between 3 and 20 (Figure 1(d)). The mutational spectra of SCLC were dominated by missense, where indel and frameshift mutations occurred predominantly in TP53, RB1, and KMT2D. There were two mutation hotspots, c.832C $>\mathrm{T}$ (p.P278S) and c.1548G > A (p.W516X), observed in TP53 and RB1, respectively (Table S2). No CNV mutations were found in any of the genes.

3.4. SCLC Gene Variant Association with Serum Biomarkers and Clinical Stage. Because NSE associated with clinical stage, we also searched among the 147 genes for those that associated with NSE levels. We found 6 genes (WT1, NOTCH1, EPHA3, KDM6A, SETD2, ACVR1B) associated with serum NSE levels $(P=0.007-0.046$; Figure 2$)$. Generally, the more mutations to these 6 genes, the higher were the NSE levels $(P=0.0016)$ compared with one or no mutation to these 6 genes that were not associated with NSE levels $(P=0.1139)$, and furthermore, these 6 genes were associated with clinical stages II + III compared to I (marginal $P=0.06$, Figure 3).

\section{Discussion}

From 32 Chinese SCLC patients, we detected 147 out of 303 cancer driver oncogenes, including three previously implicated genes, TP53, RB1, and KMT2D, that appeared in 79\% of our patients across all clinical stage. Together with $L R R K 2$, $B R C A 1, P T C H 1, A R I D 2$, and APC, these eight genes represented top $5 \%$ of all genes that occurred in $90 \%$ of patients. In this study, we further found that serum protein marker NSE significantly associated with higher clinical stages II + III $\left(P=7 \times 10^{-4}\right)$. In particular, increased mutations to 6 genes (WT1, NOTCH1, EPHA3, KDM6A, SETD2, ACVR1B) significantly associated with higher NSE levels $(P=0.0016)$ and stages II + III $(P=0.06)$.

Loss of functions to TP53 and RB1 highly associate with the pathogenesis of SCLC [27] and are found in 82\% and $62 \%$ of SCLC patients, respectively [28]. Previous study indicates that $\mathrm{G}: \mathrm{C}$ to $\mathrm{T}: \mathrm{A}$ transversions are the most frequently observed nucleotide substitutions to TP53 in lung cancer [29], which is consistent with our finding. In contrast, KMT2D mutations are less related to TP53 and RB1; it has instead been linked to a longer survival in patients with lung neuroendocrine tumors [30] despite that it has also been shown not to be involved in reducing survival of NSCLC patients [31]. In our study, the third most frequent gene mutations were in the KMT2D gene, whether the KMT2D gene plays a crucial role in survival of Chinese SCLC patient needs to be further investigated.

Among the five novel SCLC-related mutated genes found in this study, the LRRK2 is a mutation gene in the Chinese population with familial Parkinson's disease [32]. LRRK2 mutation carriers have an increased risk of cancer, especially for hormone-related cancer, and SCLC is associated with polypeptide hormone production [33]. The BRCA1 is involved in tumor suppression and homologous recombination repair in response to DNA breaks and may be a modulator of mitotic spindle assembly [34, 35]. Whole exome sequencing of the lung cancer has revealed a germline BRCA1 deficiency mutational signature [36]. We have identified mutations in LRRK2 and BRCA1 genes, and this represents the first report in the Chinese patients with SCLC. Finally, $A R I D 2$ is a tumor suppressor gene, where inactivating mutations along the ARID2 coding region is also detected in 


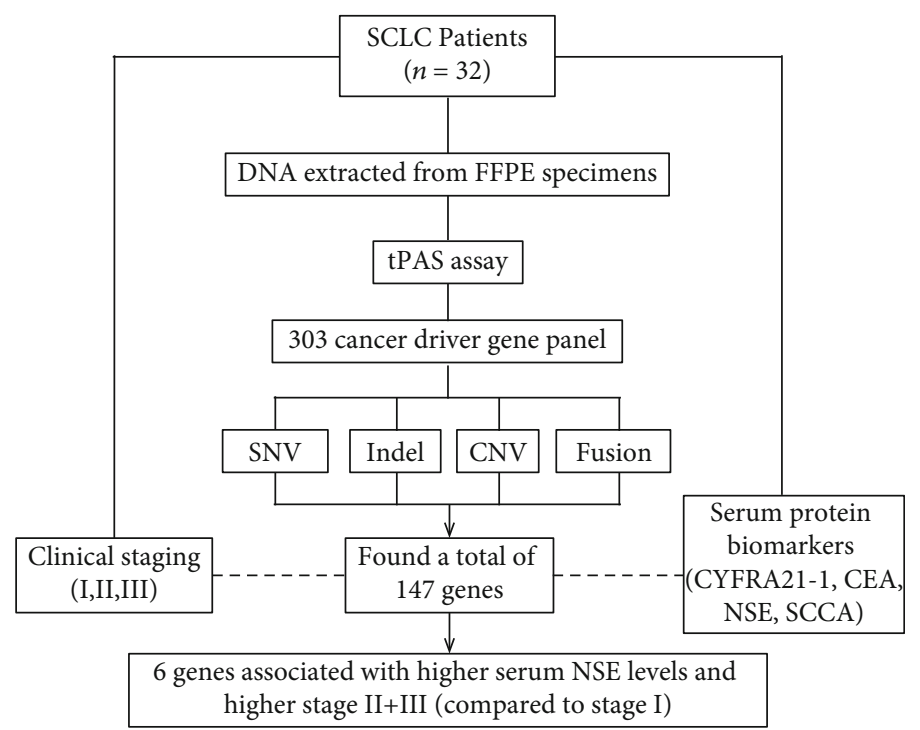

(a)
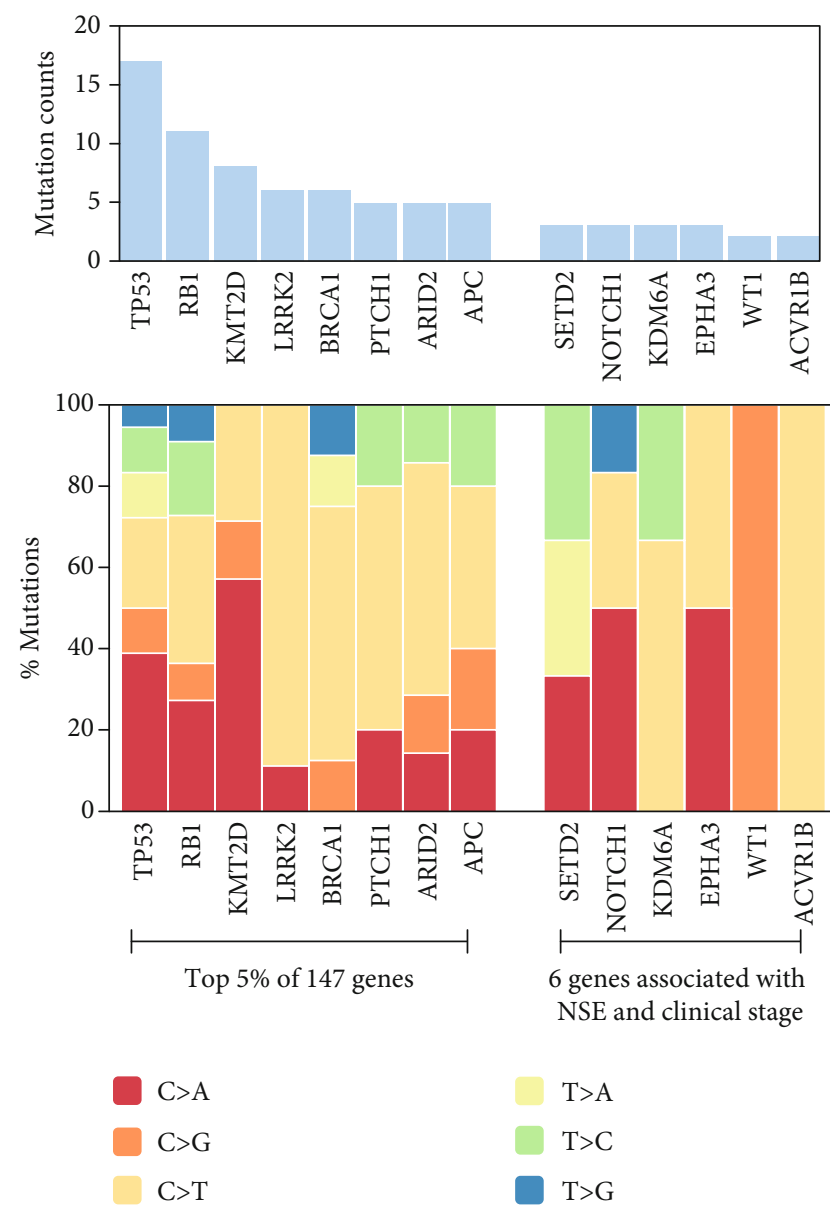

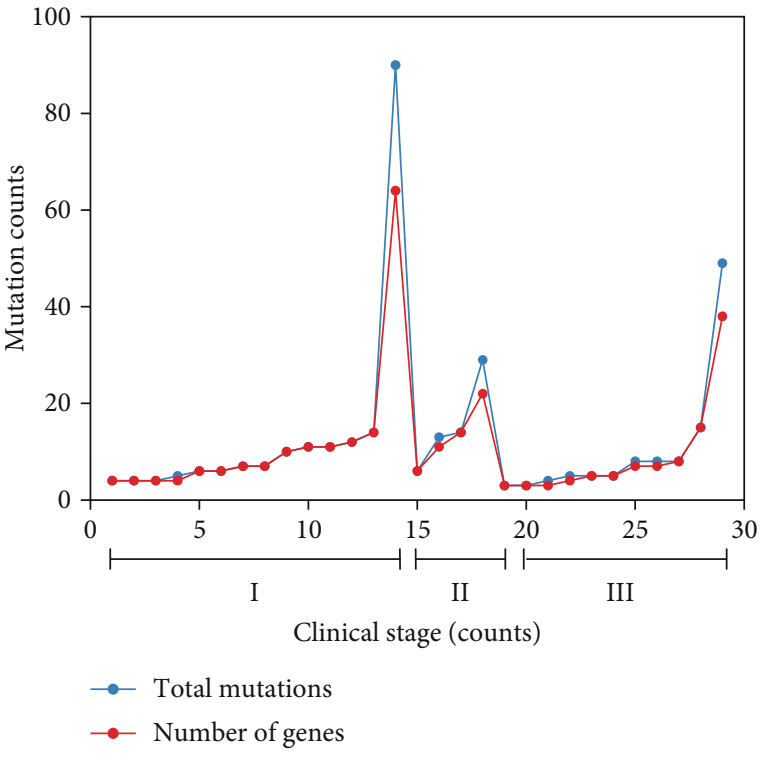

(b)

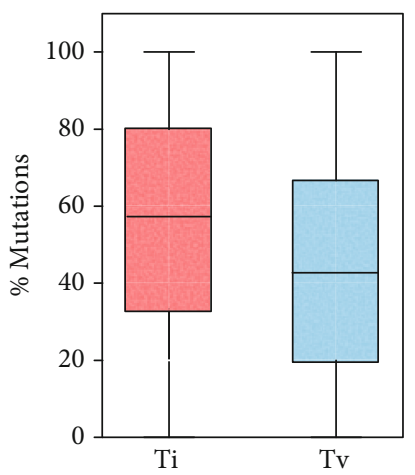

Figure 1: Continued. 


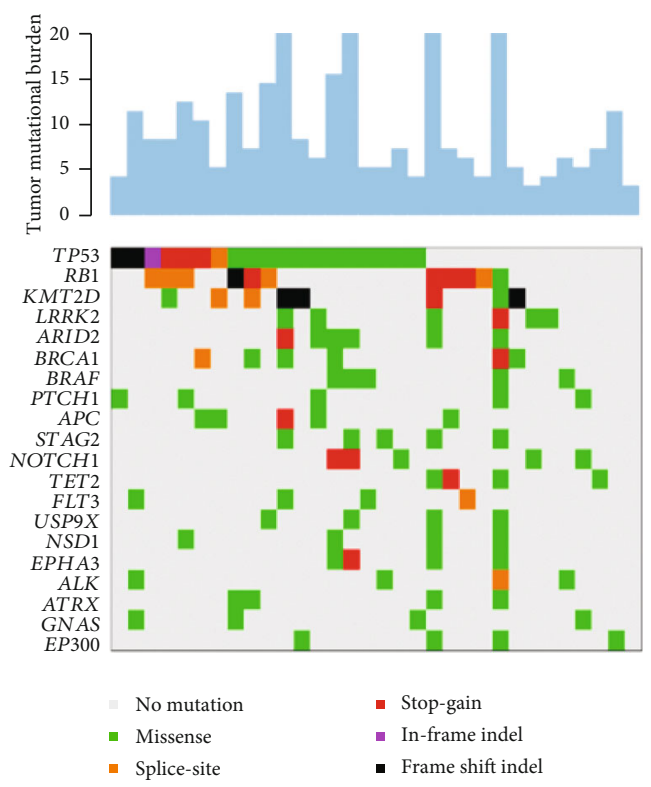

(d)

Figure 1: Study overview: (a) sequencing and analytical pipeline; (b) total mutations and number of genes per patient arranged according to clinical stages I, II, and III; (c) mutation counts of top 5\% SCLC-related mutated genes observed in 90\% of patients and 6 genes associated with NSE and clinical stage, where mutation to same gene is counted once (top) with their individual and combined summary of transition (Ti) and transversion (Tv) mutations (bottom); (d) mutational landscape of SCLC showing the number of coding somatic mutations per megabase of DNA (top) and a matrix of 20 frequently mutated genes colored by the type of variation (bottom).
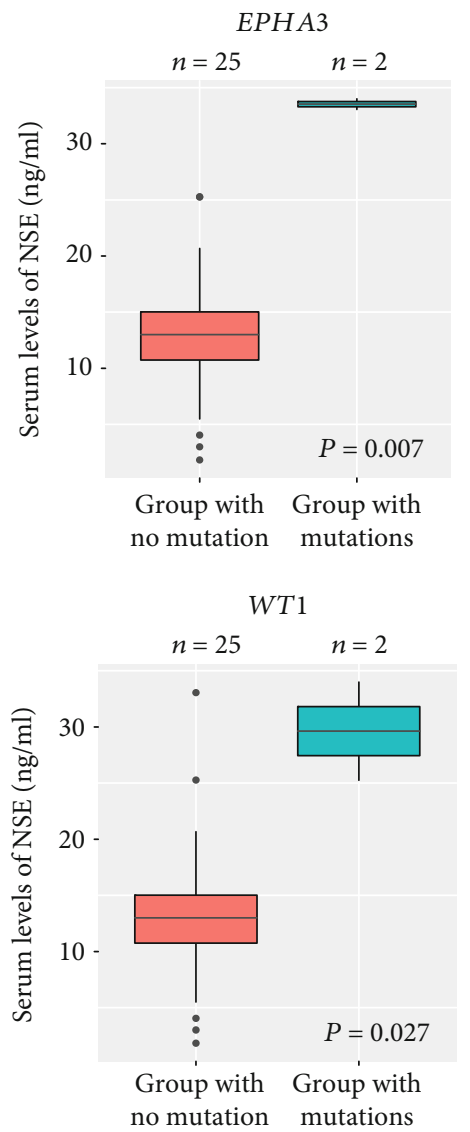
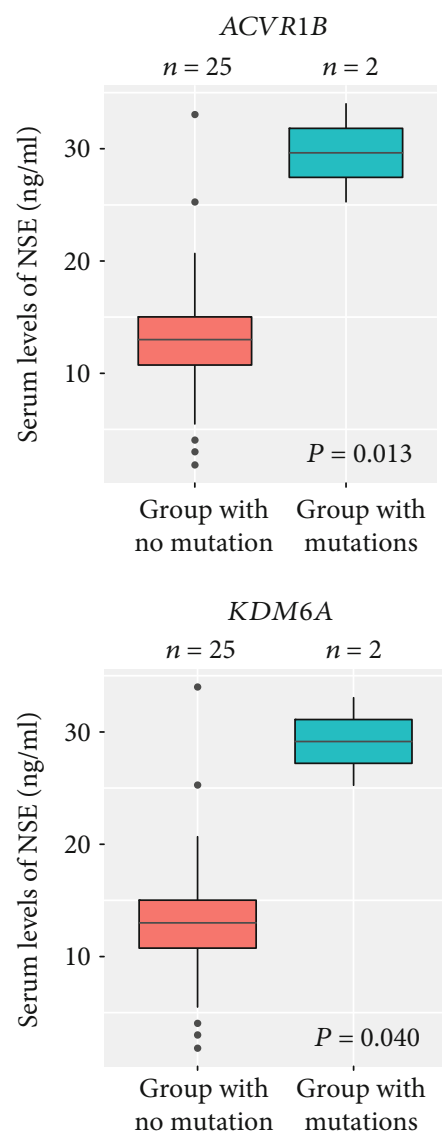
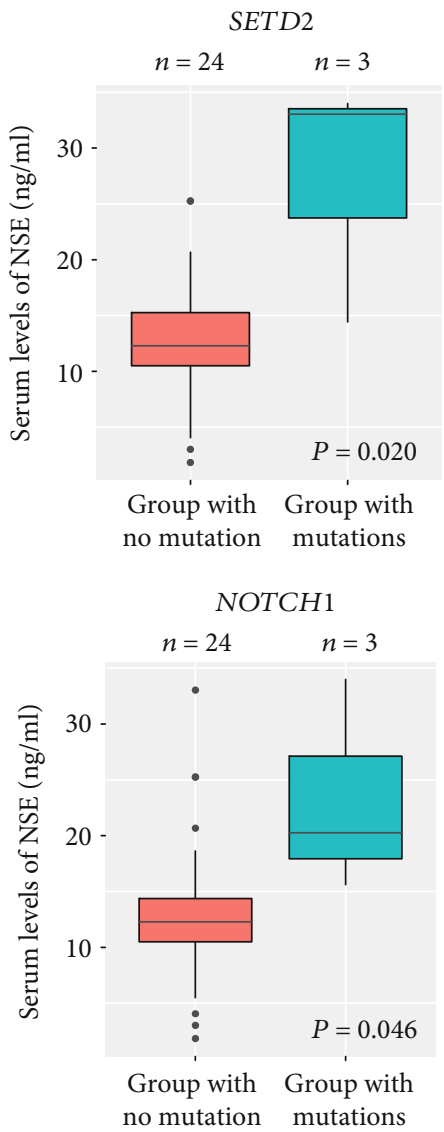

FIgURE 2: Significantly higher serum levels of NSE in SCLC patients with mutations to the following 6 genes (Wilcoxon rank sum test $P=$ $0.007-0.046)$. 


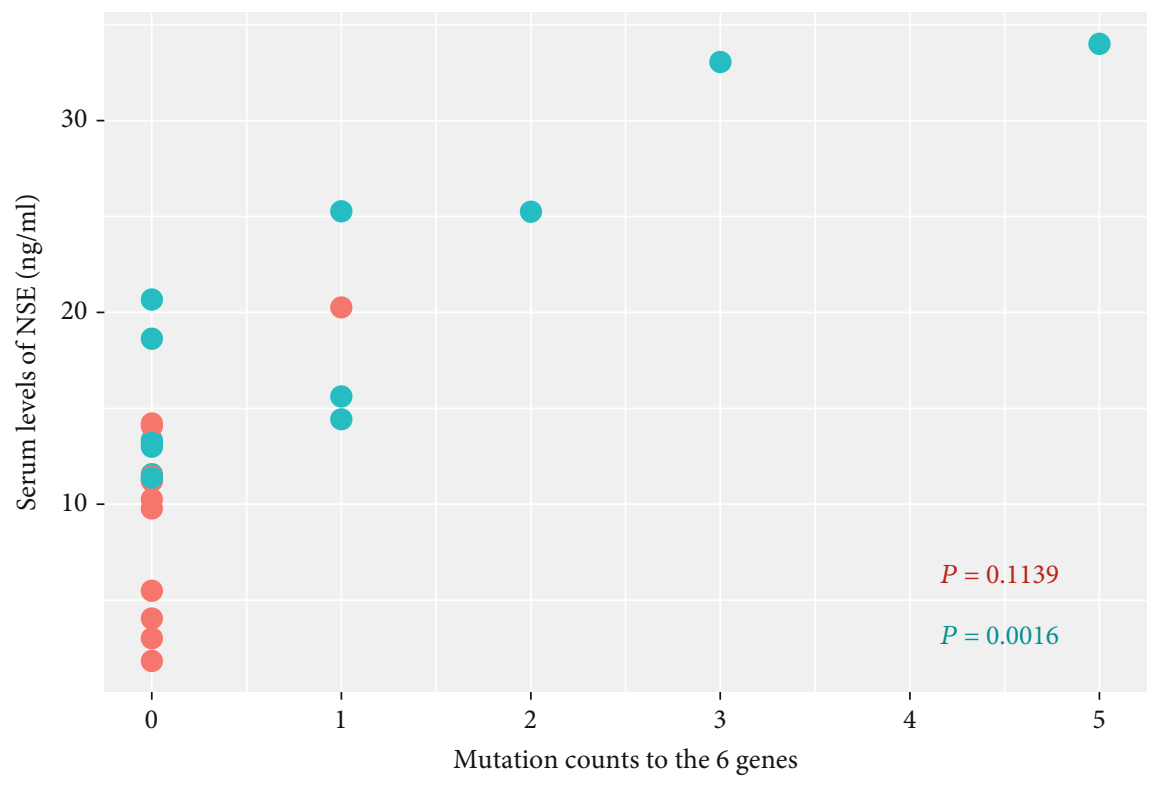

(a)

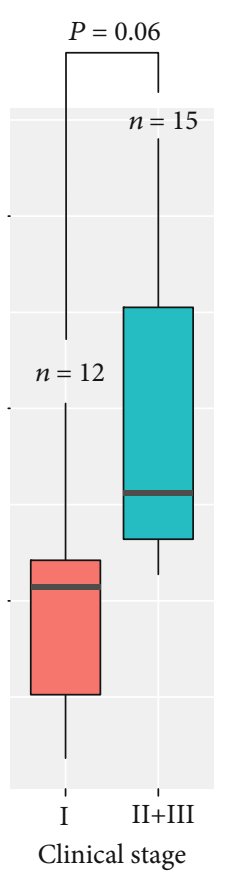

(b)

FIGURE 3: Increased mutations to 6 genes are associated with higher serum levels of NSE and clinical stage of SCLC. Scatterplot (a) shows patients with increased mutations to the following 6 genes (WT1, NOTCH1, EPHA3, KDM6A, SETD2, ACVR1B) have significantly higher NSE levels (Spearman's correlation test $P=0.0016)$ compared to those with $\leq 1$ mutation to these 6 genes $(P=0.1139)$. Boxplot $(b)$ shows patients with increased mutations to these 6 genes also have a significantly higher clinical stages II + III compared to stage I (Wilcoxon rank sum test $P=0.06$ ).

NSCLC [37]. ARID2 knockout results in dysfunction of DNA repair process, leading to susceptibility to carcinogens in human hepatocellular carcinoma cells [38]. However, it is unclear how ARID2 mutations are associated with SCLC progression. We identified a $46.9 \%$ variant allele fraction to c.3004A > G (p.T1002A) in ARID2 of a SCLC patient. To our knowledge, our data is the first to report such high frequency SNV of ARID2 gene in SCLC.

In this study, serum levels of NSE associated with higher clinical stage of SCLC. It has been reported that NSE (81.2\%) has the highest sensitivity, followed by CEA (42.7\%), CYFRA21-1 (32.3\%), and SCCA (1\%), and the average concentration of NSE was statistically higher in the patients with extensive disease (88.2\%) compared to limited disease (73.3\%) [39]. Recent studies, such as CancerSEEK, used 16 gene mutations combined with abnormal levels of 8 protein biomarkers for early diagnosis of lung cancer [40]. Similarly, we found 6 mutated genes that could associate with serum NSE to provide information about the severity of SCLC.

Some limitations of this study should be noted. First, we focused on SNV and indels as their function could be predicted by numerous methods and that heterogeneity has immediate clinical consequences for treatment selection [41]. Second, we could not evaluate recurrent noncoding, copy-number, or epigenetic mutations because the functional prediction methods for them were not yet available. Third, we did not assess micrometastases that are not visible clinically, so could not exclude those mutations in yet undiscovered driver genes of metastases. Finally, the top 5\% mutated genes did not overlap with the 6 genes associated with NSE and stage, might be the small sample size, or that 6 genes represented a subpopulation of SCLC patients.

\section{Conclusion}

About $50 \%$ of oncogenic driver mutations are detected by our panel, suggesting it is moderately enriched for SCLC detection. Apart from capturing the most important SCLCrelated mutant genes reported in other studies, we further found 5 genes and increased mutations to 6 genes associated with higher NSE and clinical stage. More investigations are required to evaluate the possibility of combining these mutant genes with NSE into a fast and simple companion diagnostic kit for clinicians to detect SCLC stage.

\section{Data Availability}

The data are available upon the authors' reasonable request.

\section{Conflicts of Interest}

The authors declare that they have no conflicts of interest.

\section{Acknowledgments}

The authors wish to thank Liu Shihong, Wang Lientu, and Chin Lihan for their contributions to this manuscript. This work was supported by grants from the National Natural Science Foundation of China $(81774078,81802886)$ and by 
grants from the Natural Science Foundation of Liaoning province, China (20180550693).

\section{Supplementary Materials}

Figure S1: serum levels of NSE significantly associated with a higher clinical stages II + III of SCLC (Wilcoxon rank sum test $\left.P=7 \times 10^{-4}\right)$. Table S1: clinical category IASLC T1-4 (any N) M0 or N0-N3 (any T) M0 SCLC. Table S2: list of 147 SCLC-related mutant genes ranked by total mutation counts. (Supplementary Materials)

\section{References}

[1] R. L. Siegel, K. D. Miller, and A. Jemal, "Cancer statistics, 2018," CA: a Cancer Journal for Clinicians, vol. 68, no. 1, pp. 7-30, 2018.

[2] National Lung Screening Trial Research Team, D. R. Aberle, A. M. Adams et al., "Reduced lung-cancer mortality with lowdose computed tomographic screening," The New England Journal of Medicine, vol. 365, no. 5, pp. 395-409, 2011.

[3] A. Sharp, J. Bhosle, F. Abdelraouf, S. Popat, M. O'Brien, and T. A. Yap, "Development of molecularly targeted agents and immunotherapies in small cell lung cancer," European Journal of Cancer, vol. 60, pp. 26-39, 2016.

[4] S. N. Waqar and D. Morgensztern, "Treatment advances in small cell lung cancer (SCLC)," Pharmacology \& Therapeutics, vol. 180, pp. 16-23, 2017.

[5] M. C. Pietanza, L. A. Byers, J. D. Minna, and C. M. Rudin, "Small cell lung cancer: will recent progress lead to improved outcomes?" Clinical Cancer Research, vol. 21, no. 10, pp. 2244-2255, 2015.

[6] F. A. Shepherd, R. J. Ginsberg, R. Feld, W. K. Evans, and E. Johansen, "Surgical treatment for limited small-cell lung cancer: The University of Toronto Lung Oncology Group experience," The Journal of Thoracic and Cardiovascular Surgery, vol. 101, no. 3, pp. 385-393, 1991.

[7] E. F. Patz Jr., M. J. Campa, E. B. Gottlin, I. Kusmartseva, X. R. Guan, and J. E. Herndon II, "Panel of serum biomarkers for the diagnosis of lung cancer," Journal of Clinical Oncology : Official Journal of the American Society of Clinical Oncology, vol. 25, no. 35, pp. 5578-5583, 2007.

[8] H. Wang, T. Shi, W. J. Qian et al., "The clinical impact of recent advances in LC-MS for cancer biomarker discovery and verification," Expert Review of Proteomics, vol. 13, no. 1, pp. 99-114, 2016.

[9] L. A. Liotta and E. F. Petricoin 3rd, "The promise of proteomics," Clinical Advances in Hematology \& Oncology, vol. 1, no. 8, pp. 460-462, 2003.

[10] O. Fiala, M. Pesek, J. Finek et al., "Prognostic significance of serum tumor markers in patients with advanced-stage NSCLC treated with pemetrexed-based chemotherapy," Anticancer Research, vol. 36, no. 1, pp. 461-466, 2016.

[11] J. D. Cohen, A. A. Javed, C. Thoburn et al., "Combined circulating tumor DNA and protein biomarker-based liquid biopsy for the earlier detection of pancreatic cancers," Proceedings of the National Academy of Sciences of the United States of America, vol. 114, no. 38, pp. 10202-10207, 2017.

[12] Y. Zhang and J. He, "The development of targeted therapy in small cell lung cancer," Journal of Thoracic Disease, vol. 5, no. 4, pp. 538-548, 2013.
[13] M. Lucchi, A. Mussi, G. Fontanini, P. Faviana, A. Ribechini, and C. A. Angeletti, "Small cell lung carcinoma (SCLC): the angiogenic phenomenon," European Journal of CardioThoracic Surgery, vol. 21, no. 6, pp. 1105-1110, 2002.

[14] M. Peifer, L. Fernández-Cuesta, M. L. Sos et al., "Integrative genome analyses identify key somatic driver mutations of small- cell lung cancer," Nature Genetics, vol. 44, no. 10, pp. 1104-1110, 2012.

[15] T. Takahashi, M. Nau, I. Chiba et al., "p53: a frequent target for genetic abnormalities in lung cancer," Science, vol. 246, no. 4929, pp. 491-494, 1989.

[16] I. I. Wistuba, A. F. Gazdar, and J. D. Minna, "Molecular genetics of small cell lung carcinoma," Seminars in Oncology, vol. 28, 2 Supplement 4, pp. 3-13, 2001.

[17] N. Mori, J. Yokota, T. Akiyama et al., "Variable mutations of the RB gene in small-cell lung carcinoma," Oncogene, vol. 5, no. 11, pp. 1713-1717, 1990.

[18] A. Augert, Q. Zhang, B. Bates et al., "Small cell lung cancer exhibits frequent inactivating mutations in the histone methyltransferase KMT2D/MLL2: CALGB 151111 (Alliance)," Journal of Thoracic Oncology, vol. 12, no. 4, pp. 704-713, 2017.

[19] S. A. Forbes, D. Beare, P. Gunasekaran et al., "COSMIC: exploring the world's knowledge of somatic mutations in human cancer," Nucleic Acids Research, vol. 43, no. D1, pp. D805-D811, 2015.

[20] The Cancer Genome Atlas Research Network, J. N. Weinstein, E. A. Collisson et al., "The Cancer Genome Atlas pan-cancer analysis project," Nature Genetics, vol. 45, no. 10, pp. 11131120, 2013.

[21] K. Zhang and H. Wang, "Cancer Genome Atlas pan-cancer analysis project," Zhongguo Fei Ai Za Zhi, vol. 18, no. 4, pp. 219-223, 2015.

[22] L. Ye, H. Li, F. Zhang, T. Lv, H. Liu, and Y. Song, "Expression of KIF23 and its prognostic role in non-small cell lung cancer: analysis based on the data-mining of Oncomine," Zhongguo Fei Ai Za Zhi, vol. 20, no. 12, pp. 822-826, 2017.

[23] A. McKenna, M. Hanna, E. Banks et al., "The Genome Analysis Toolkit: a MapReduce framework for analyzing nextgeneration DNA sequencing data," Genome Research, vol. 20, no. 9, pp. 1297-1303, 2010.

[24] K. Cibulskis, M. S. Lawrence, S. L. Carter et al., "Sensitive detection of somatic point mutations in impure and heterogeneous cancer samples," Nature Biotechnology, vol. 31, no. 3, pp. 213-219, 2013.

[25] K. Wang, M. Li, and H. Hakonarson, “ANNOVAR: functional annotation of genetic variants from high-throughput sequencing data," Nucleic Acids Research, vol. 38, no. 16, article e164, 2010.

[26] W. Zhou, T. Chen, Z. Chong et al., "TransVar: a multilevel variant annotator for precision genomics," Nature Methods, vol. 12, no. 11, pp. 1002-1003, 2015.

[27] J. George, J. S. Lim, S. J. Jang et al., "Comprehensive genomic profiles of small cell lung cancer," Nature, vol. 524, no. 7563, pp. 47-53, 2015.

[28] L. Jiang, J. Huang, B. W. Higgs et al., "Genomic landscape survey identifies SRSF1 as a key oncodriver in small cell lung cancer," PLoS Genetics, vol. 12, no. 4, article e1005895, 2016.

[29] M. Hollstein, D. Sidransky, B. Vogelstein, and C. C. Harris, "p53 mutations in human cancers," Science, vol. 253, no. 5015, pp. 49-53, 1991. 
[30] M. Simbolo, A. Mafficini, K. O. Sikora et al., "Lung neuroendocrine tumours: deep sequencing of the four World Health Organization histotypes reveals chromatin-remodelling genes as major players and a prognostic role for TERT, RB1, MEN1 and KMT2D," The Journal of Pathology, vol. 241, no. 4, pp. 488-500, 2017.

[31] F. Ardeshir-Larijani, P. Bhateja, M. B. Lipka, N. Sharma, P. Fu, and A. Dowlati, "KMT2D mutation is associated with poor prognosis in non-small-cell lung cancer," Clinical Lung Cancer, vol. 19, no. 4, pp. e489-e501, 2018.

[32] L. Wang, J. F. Guo, L. L. Nie et al., "A novel LRRK2 mutation in a mainland Chinese patient with familial Parkinson's disease," Neuroscience Letters, vol. 468, no. 3, pp. 198-201, 2010.

[33] K. Havemann, W. Luster, C. Gropp, and R. Holle, "Peptide hormone production associated with small cell lung cancer," Recent Results in Cancer Research, vol. 97, pp. 65-76, 1985.

[34] L. V. Lotti, L. Ottini, C. D'Amico et al., "Subcellular localization of the BRCA1 gene product in mitotic cells," Genes, Chromosomes \& Cancer, vol. 35, no. 3, pp. 193-203, 2002.

[35] P. B. Mullan, J. E. Quinn, P. M. Gilmore et al., "BRCA1 and GADD45 mediated G2/M cell cycle arrest in response to antimicrotubule agents," Oncogene, vol. 20, no. 43, pp. 6123-6131, 2001.

[36] S. Cedrés, E. Felip, C. Cruz et al., “Activity of HSP90 inhibiton in a metastatic lung cancer patient with a germline BRCA1 mutation," Journal of the National Cancer Institute, vol. 110, no. 8, pp. 914-917, 2018.

[37] G. Manceau, E. Letouzé, C. Guichard et al., "Recurrent inactivating mutations of ARID2 in non-small cell lung carcinoma," Journal International Du Cancer, vol. 132, no. 9, pp. 22172221, 2013.

[38] A. Oba, S. Shimada, Y. Akiyama et al., "ARID2 modulates DNA damage response in human hepatocellular carcinoma cells," Journal of Hepatology, vol. 66, no. 5, pp. 942-951, 2017.

[39] R. Molina, J. M. Auge, J. M. Escudero et al., "Mucins CA 125, CA 19.9, CA 15.3 and TAG-72.3 as tumor markers in patients with lung cancer: comparison with CYFRA 21-1, CEA, SCC and NSE," Tumour Biology, vol. 29, no. 6, pp. 371-380, 2008.

[40] J. D. Cohen, L. Li, Y. Wang et al., "Detection and localization of surgically resectable cancers with a multi-analyte blood test," Science, vol. 359, no. 6378, pp. 926-930, 2018.

[41] B. Vogelstein, N. Papadopoulos, V. E. Velculescu, S. Zhou, L. A. Diaz, and K. W. Kinzler, "Cancer genome landscapes," Science, vol. 339, no. 6127, pp. 1546-1558, 2013. 